\title{
The effect on critical velocity of runnings with change of direction in soccer
}

\author{
Authors' Contribution: \\ A Study Design \\ B Data Collection \\ C Statistical Analysis \\ D Data Interpretation \\ E Manuscript Preparation \\ F Literature Search \\ Erdal Ari ${ }^{1 A B C D}$, Hamit Cihan ${ }^{2 A B C D}$, Abdullah Çetindemir ${ }^{2 A B C D}$ \\ ${ }^{1}$ Physical Education and Sports School, Ordu University, Ordu, Turkey \\ ${ }^{2}$ Faculty of Sports Sciences, Trabzon University, Akçaabat, Turkey \\ G Funds Collection
}

\section{abstract}

Background: The aim of this study was to examine relationships between Yo-Yo intermittent recovery level 1 test (YYIR1T) performance and critical velocity determined by test protocols consisting of runs with a change of direction and straight runs.

Material and methods:

Twelve young soccer players voluntarily participated in study (age: $17.07 \pm 0.24$ years, training experience: $8.42 \pm 2.50$ years, height: $178.58 \pm 5.76 \mathrm{~cm}$, weight: $70.67 \pm 6.14 \mathrm{~kg}$, body mass index: 22.16 \pm 1.59 ). To determine critical velocity, 6-, 9- and 12-minute runs were performed with maximum effort on a straight-line running track and a running track with a change of direction. The critical velocity was determined by two linear regression models. YYIR1T was performed to determine players' aerobic endurance. The critical velocity value of the two test tracks was compared by the paired samples T-test. The correlation between test parameters was determined by Spearman's correlation coefficient.

Results: A significant difference between anaerobic distance capacity and the mean running speed of the two test tracks $(p<0.05)$ was found. There was a significant correlation between critical velocity in the straight-line test track and the YYIR1T performance $(p<0.05)$.

Conclusions: Consequently, it might be said that runs with a change of direction might increase energy expenditure and anaerobic distance capacity was highly affected by those compared to critical velocity.

Key words: critical velocity, Yo-Yo intermittent recovery test, aerobic endurance, change of direction, soccer.

\section{article details}

Article statistics: Word count: 4,302; Tables: 3; Figures: 2; References: 41

Received: January 2021; Accepted: June 2021; Published: September 2021

Full-text PDF: http://www.balticsportscience.com

Copyright @ Gdansk University of Physical Education and Sport, Poland

Indexation: Celdes, Clarivate Analytics Emerging Sources Citation Index (ESCI), CNKI Scholar (China National Knowledge Infrastructure), CNPIEC, DOAJ, EBSCO - Central \& Eastern European Academic Source, EBSCO - SPORTDiscus, EBSCO Discovery Service, Google Scholar, Index Copernicus, J-Gate, Naviga (Softweco, Primo Central (ExLibris), ProQuest - Family Health, ProQuest - Health \& Medical Complete, ProQuest - Illustrata: Health Sciences, ProQuest Nursing \& Allied Health Source, Summon (Serials Solutions/ProQuest, TDOne (TDNet), Ulrich's Periodicals Directory/ ulrichsweb, WorldCat (OCLC)

Funding: This research received no specific grant from any funding agency in the public, commercial, or not-for-profit sectors.

Conflict of interests: Corresponding author:

Open Access License: Authors have declared that no competing interest exists.

Erdal ARI, Ordu University Physical Education ans Sports School, 52200, Ordu, TURKEY. Telephone: +90 5325691852 , E-mail: arierdal@hotmail.com

This is an open access article distributed under the terms of the Creative Commons Attribution-Non-Commercial-NoDerivatives 4.0 International (https://creativecommons.org/licenses/by-nc-nd/4.0/), which permits use, distribution, and reproduction in any medium, provided the original work is properly cited, the use is non-commercial and is otherwise in compliance with the license. 


\section{INTRODUCTION}

The endurance capacity is an important physical fitness parameter in soccer. A soccer game lasts 90 minutes. The game includes many physical activities, such as jogging, standing, backward running, walking, low intensity running. These activities mainly depend on aerobic players' endurance capacity. However, there are also anaerobic activities in soccer game, such as high intensity running, jumps, tackling, sprints, dribbling, accelerating and turns. These anaerobic activities cause lactate accumulation in soccer players. The phosphorylation of glycogen (glycogenolysis) increases during anaerobic activities. The lactate can be eliminated from blood with low intensity (aerobic) exercises. The low intensity activities are performed to eliminate lactate from the active muscle groups [1]. Therefore, high aerobic capacity contributes to players' performance. It was determined that elite soccer players covered 11 kilometers' distance in a soccer match [2]. The maximum oxygen uptake $\left(\mathrm{VO}_{2} \max \right)$ is one of the factors determining the distance covered in a soccer match. Therefore, $\mathrm{VO}_{2}$ max is a parameter used in order to increase and evaluate soccer players' physical fitness level [3].

The $\mathrm{VO}_{2}$ max and aerobic endurance capacity can be determined with various measurement methods. There are some indicators of aerobic endurance. Critical velocity (CV) is an important indicator of aerobic endurance. CV is known as the highest running velocity without fatigue in prolonged exercises used dominantly by the aerobic system [4]. Firstly, Monod and Scherer [5] defined critical power concept in a cycle ergometer. Then, the critical power concept was adapted to running exercises by Ettema et al. [6]. In the critical power concept, the anaerobic work capacity is known as an amount of work performed by anaerobic energy stores above the critical power value [7]. There was a linearity between the total work (covered distance for critical velocity) and exercise time in the critical power concept [5]. In a linear relationship, the slope coefficient was critical power (critical velocity) and the y-intercept was anaerobic work capacity (anaerobic distance capacity for critical velocity) [8]. CV was determined by running tests on a track and a treadmill. The tests consist of two and more runs performed at different times. The critical power (or critical velocity) may be described as the highest rate of ATP synthesis with using oxygen without anaerobic energy reserves for energy production [9]. The aerobic endurance indicates the capacity of ATP production without lactate accumulation in the presence of oxygen. Therefore, $\mathrm{VO}_{2} \max$ is an important indicator of endurance capacity and resistance to fatigue. The oxygen uptake capacity is the most important factor determining critical power (or critical velocity) [10].

Soccer dominantly includes aerobic activities. However, high intensity anaerobic activities (turns, acceleration, jumps, intermittent activities, sprints) are alternately performed with aerobic activities (standing, walking and jogging) [11]. Also, actions with a change of direction are frequently performed in soccer game. The high aerobic endurance increases tolerance to fatigue and decreases the time of recovery. Intermittent anaerobic activity performance may be developed by increasing lactate tolerance depending on aerobic endurance. Thus, soccer players may frequently perform intermittent high intensity activities during game. The aerobic endurance of soccer players must be measured and evaluated with performance tests to reach optimal performance. CV is a useful and easy measurement tool of aerobic performance in soccer. Expensive test equipment is not needed for CV tests. Aerobic performance may be easily determined with running tests. $\mathrm{CV}$ is recommended for measurement of aerobic endurance performance in soccer players [12]. Lord et al. [13] found a relationship between the maximal mean speed values at soccer matches and CV. Similarly, it was indicated that CV was a non-invasive indicator of endurance performance in soccer players [14]. The endurance training sessions of soccer players may be planned and monitored by coaches with CV. CV has practical and simple test protocols. Therefore, the CV is a practical parameter for measuring aerobic endurance performance in soccer. In this context, the aim of the present study was to examine 
relationships between Yo-Yo intermittent recovery level 1 test (YYIR1T) performance and a CV parameter determined by test protocols consisting of runs with a change of direction and straight runs.

\section{MATERIAL AND METHODS}

\section{Participants}

Twelve amateur young soccer players voluntarily participated in the study (Age: $17.07 \pm 0.24$ years, training experience: $8.42 \pm 2.50$ years, height: $178.58 \pm 5.76 \mathrm{~cm}$, weight: $70.67 \pm 6.14$ $\mathrm{kg}$, body mass index: $22.16 \pm 1.59$ ). The subjects consisted of players playing soccer in the U-17 category of the team in Turkey Super League, and many of them were players of youth national teams. All players were informed about the study protocol. Informed voluntary consent form was filled in by all players. The study was performed in line with principles of the Helsinki Declaration.

\section{Data Collection}

Straight-Line Running Tests (SLRT) and Running Tests With a Change of Direction (RTCD) Both SLRT and RTCD consisted of three different running tests (6-, 9-, and 12-minute running tests). All tests were performed at the same time of day with 48 hours rest interval. The 6-, 9and 12-minute runs were performed with maximal effort in both straight-line track and track with a change of direction on a synthetic grass soccer pitch (Figure 1). The running tracks were marked by cones using a portable steel meter. During three days before tests, vigorous exercise was not performed. The players performed warm-up and stretching exercises before all running tests. The players tried to cover the maximum distance in 6-, 9- and 12-minute running tests. Each test duration was measured with a hand chronometer (Casio HS-70W1DF, Tokyo, Japan). The covered distance values during tests were determined in meter unit. The heart rate (HR) value during the tests was automatically recorded by heart rate monitors (V800, Polar Electro OY, Kempele, Finland). The covered distance and test duration were manually recorded to determine the CV and anaerobic distance capacity (ADC) parameters. The mean running speed of three tests (MRST) and the mean of the maximum heart rates in tests (MMHRT) were also determined as test parameters.

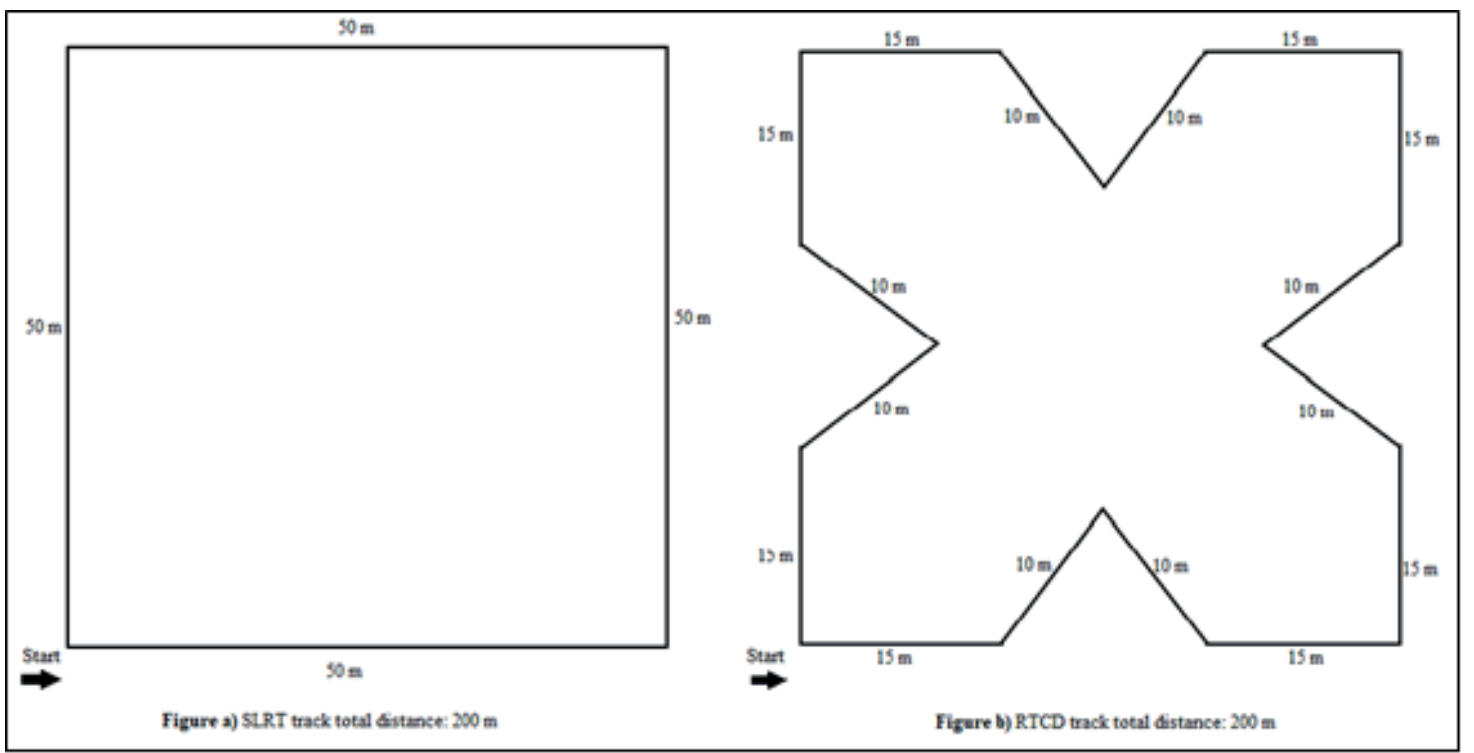

Fig. 1. The Tracks of Straight-Line Running Tests (SLRT) and Running Tests with a Change of Direction (RTCD) 
Yo-Yo Intermittent Recovery Level 1 Test (YYIR1T)

The YYIR1T was used to determine aerobic endurance of soccer players. The YYIR1T was performed on a synthetic grass soccer pitch after one week from running tests. The YYIR1T was performed at same time of day as SLRT and RTCD. During three days before test, vigorous exercise was not performed. The YYIR1T consists of a 20-m running track and 5-m active recovery area [15] (Figure 2). The running speed of YYIR1T is periodically increased by audio signals. The audio signal of YYIR1T was emitted by a portable CD player (Philips Az1030, Eindhoven, Holland). 2x20 m (40 m) running and 2x5 m (10 m) active recovery running (jogging) were performed in the tests. Active recovery running $(2 \times 5 \mathrm{~m})$ was performed in a $10-\mathrm{m}$ area during 10 seconds after $2 \times 20 \mathrm{~m}$ runs. The audio signals indicate running speeds of test. The athletes adjust their running speeds, and the audio signal is tried to be caught in $20 \mathrm{~m}$ cones. The test protocol was explained to all players. After warm-up and stretching, all players performed the test. The YYIR1T began at $10 \mathrm{~km} / \mathrm{h}$ running speed. After the start of the test, $4 \times 40 \mathrm{~m}$ distance at $10-13 \mathrm{~km} / \mathrm{h}$ running speed interval and $7 \times 40 \mathrm{~m}$ distance at 13.5-14 km/h running speed interval were covered. Then, the running speed of the test increased by $0.5 \mathrm{~km} / \mathrm{h}$ after every $8 \times 40 \mathrm{~m}$ running bout. The test ended when the audio signal was not caught in $20 \mathrm{~m}$ cones or athletes left the test voluntarily. The final running speed of the test was defined as the maximum aerobic speed (MAS). The covered distance and MAS in YYIR1T were manually recorded in a test form. The HR value of the test was automatically recorded by heart rate monitors (V800, Polar Electro OY, Kempele, Finland). The VO2max value of players was indirectly calculated according to a formula of Bangsbo et al. [16]:

$\mathrm{VO}_{2} \max (\mathrm{ml} / \mathrm{kg} / \mathrm{min})=$ Covered distance in YYIR1T $(\mathrm{m}) \times 0.0084+36.4$

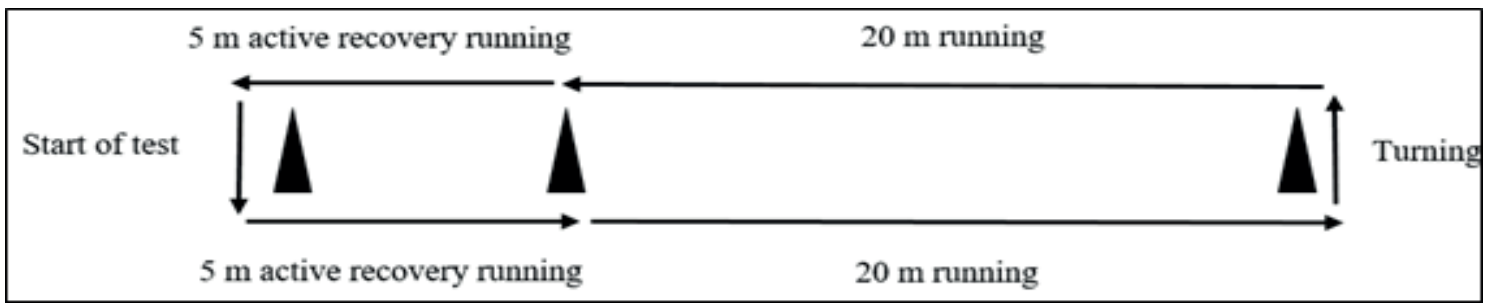

Fig. 2. Yo-Yo Intermittent Recovery Level 1 Test (YYIR1T) Track

\section{Determination of Critical Velocity Parameters}

Two linear mathematical models were used to determine CV parameters. The 6-, 9- and 12-minute running parameters of each soccer player were used in determination of CV parameters [17]. The CV and ADC parameters were determined by mathematical models using the covered distance, running duration and running speed values of 6,- 9- and 12-minute runs in both SLRT and RTCD. The CV and ADC parameters of SLRT and RTCD were calculated separately with mathematical models. These mathematical models consists of linear regression models. The first mathematical model was the linear total distance (LinTD). The Lin-TD model consists of linear regression analysis between the covered distance (D) and the running duration (t) in 6-, 9- and 12-minute running tests [5, 18-24]:

$$
\mathrm{D}=\mathrm{ADC}+\mathrm{CV} \times \mathrm{t}
$$

The regression slope of the Lin-TD model was the CV parameter. The y-intercept of the regression line in the model was the $\mathrm{ADC}$ parameter.

The linear velocity model (Lin-V) was used as the second linear regression model to determine $\mathrm{CV}$ and $\mathrm{ADC}$ parameters. The $\mathrm{CV}$ and ADC parameters were derived by linear regression 
analysis between running velocity $(\mathrm{V})$ and the inverse of running durations (1/t) values of 6-, 9- and 12-minute running tests in the Lin-V model [7, 19, 21-22, 24-29]:

$$
\mathrm{V}=\mathrm{ADC} \times(1 / \mathrm{t})+\mathrm{CV}
$$

The CV parameter was derived from y-intercept of the regression line in the Lin-V model. The ADC parameter was obtained from the slope of the regression line in the model.

\section{Data Analysis}

The study data was presented as descriptive statistics ( $\overline{\mathrm{X}} \pm \mathrm{SD})$. The CV and ADC parameters of each player were determined using Lin-TD and Lin-V linear regression models. The linear regression models were computed in Microsoft Excel program (Version 2016, Microsoft Corp., Redmond, USA). The data distribution was examined by Shapiro-Wilk test. The SLRT and RTCD parameters were compared by a paired sample T-test. The relationship between YYIR1T parameters and SLRT and RTCD parameters was determined by Spearman correlation analysis. The study data was analyzed in the SPSS package program (IBM SPSS 22.0. Armonk, NY: IBM Corp.). The significance level in statistical analysis was set at $\mathrm{p}<0.05$.

\section{RESULTS}

The results of SLRT, RTCD and YYIR1T were presented in Table 1. It was determined that the CV value of RTCD was higher than SLRT. However, it was determined that the ADC and MRST values of SLRT were higher than RTCD.

Table 1. The Descriptive Statistics of Test Parameters in Young Soccer Players

\begin{tabular}{|c|c|c|c|}
\hline Test & Test Parameters & Mean \pm Std. Dev. & Min.-Max. \\
\hline \multirow{6}{*}{ SLRT $(n=12)$} & MMHRT (beat/min) & $192.06 \pm 8.53$ & 179.33-208.33 \\
\hline & MRST (km/h) & $13.79 \pm 1.04$ & $12.10-15.81$ \\
\hline & Lin-TD CV (km/h) & $10.77 \pm 0.84$ & $9.61-11.99$ \\
\hline & Lin-TD ADC(km) & $0.42 \pm 0.10$ & $0.28-0.60$ \\
\hline & Lin-V CV (km/h) & $10.73 \pm 1.17$ & $9.14-12.53$ \\
\hline & Lin-V ADC (km) & $0.43 \pm 0.09$ & $0.26-0.56$ \\
\hline \multirow{6}{*}{ RTCD $(n=12)$} & MMHRT (beat/min) & $193.83 \pm 8.29$ & $179.33-208.00$ \\
\hline & MRST (km/h) & $12.91 \pm 0.71$ & $12.00-14.14$ \\
\hline & Lin-TD CV (km/h) & $10.92 \pm 1.08$ & $9.00-12.64$ \\
\hline & Lin-TD ADC(km) & $0.28 \pm 0.12$ & $0.17-0.50$ \\
\hline & Lin-V CV (km/h) & $11.04 \pm 1.19$ & $8.68-12.92$ \\
\hline & Lin-V ADC (km) & $0.26 \pm 0.13$ & $0.14-0.51$ \\
\hline \multirow{4}{*}{ YYIR1T $(n=12)$} & $\mathrm{VO}_{2} \max (\mathrm{ml} / \mathrm{kg} / \mathrm{min})$ & $49.78 \pm 3.05$ & $45.14-56.56$ \\
\hline & MAS (km/h) & $15.88 \pm 0.53$ & $15.00-17.00$ \\
\hline & Distance (m) & $1593.33 \pm 363.75$ & $1040.00-2400.00$ \\
\hline & HR (beat/min) & $194.92 \pm 12.09$ & $176.00-220.00$ \\
\hline
\end{tabular}

SLRT: Straight-line running tests, RTCD: Running tests with a change of direction, YYIR1T: Yo-Yo intermittent recovery level 1 test, MMHRT: Mean of the maximum heart rates in three tests, MRST: Mean running speed of three tests, Lin-TD CV: Critical velocity in the linear total distance model, Lin-TD ADC: Anaerobic distance capacity in the linear total distance model, Lin-V CV: Critical velocity in the linear velocity model, Lin-V ADC: Anaerobic distance capacity in the linear velocity model, $\mathrm{VO}_{2}$ max: Maximum oxygen uptake, MAS: Maximum aerobic speed, HR: Heart rate after the test.

The comparison of SLRT and RTCD values was presented in Table 2. A significant difference was found between SLRT and RTCD in terms of MRST and ADC values $(p<0.05)$. There was no significant difference between SLRT and RTCD with regard to MMHRT and CV values. 
Table 2. The analysis results of the difference between straight-line running tests (SLRT) and running tests with change of direction (RTCD) in young soccer players $(n=12)$

\begin{tabular}{|c|c|c|c|c|}
\hline Parameter & Test & Mean \pm Std. Dev. & $\mathrm{t}$ & $p$ \\
\hline \multirow{2}{*}{ MMHRT (beat/min) } & SLRT & $192.06 \pm 8.53$ & \multirow{2}{*}{-1.749} & \multirow{2}{*}{0.108} \\
\hline & RTCD & $193.83 \pm 8.29$ & & \\
\hline \multirow{2}{*}{ MRST (km/h) } & SLRT & $13.79 \pm 1.04$ & \multirow{2}{*}{5.987} & \multirow{2}{*}{$0.001 *$} \\
\hline & RTCD & $12.91 \pm 0.71$ & & \\
\hline \multirow{2}{*}{ Lin-TD CV (km/h) } & SLRT & $10.77 \pm 0.84$ & \multirow{2}{*}{-0.547} & \multirow{2}{*}{0.596} \\
\hline & RTCD & $10.92 \pm 1.08$ & & \\
\hline \multirow{2}{*}{ Lin-TD ADC(km) } & SLRT & $0.42 \pm 0.10$ & \multirow{2}{*}{3.404} & \multirow{2}{*}{$0.006^{*}$} \\
\hline & RTCD & $0.28 \pm 0.12$ & & \\
\hline \multirow{2}{*}{ Lin-V CV (km/h) } & SLRT & $10.73 \pm 1.17$ & \multirow{2}{*}{-0.750} & \multirow{2}{*}{0.469} \\
\hline & RTCD & $11.04 \pm 1.19$ & & \\
\hline \multirow{2}{*}{ Lin-V ADC (km) } & SLRT & $0.43 \pm 0.09$ & \multirow{2}{*}{3.207} & \multirow{2}{*}{$0.008 *$} \\
\hline & RTCD & $0.26 \pm 0.13$ & & \\
\hline
\end{tabular}

${ }^{*} p<0.05$ SLRT: Straight-line running tests, RTCD: Running tests with a change of direction, MMHRT: Mean of the maximum heart rates in three tests, MRST: Mean running speed of three tests, Lin-TD CV: Critical velocity in the linear total distance model, Lin-TD ADC: Anaerobic distance capacity in the linear total distance model, Lin-V CV: Critical velocity in the linear velocity model, Lin-V ADC: Anaerobic distance capacity in the linear velocity model.

The correlation between SLRT and RTCD with YYIR1T was presented in Table 3. The MMHRT value of SLRT significantly correlated with the HR value of YYIR1T $(p<0.05)$. The $\mathrm{VO}_{2}$ max and distance values of YYIR1T had a significant and high correlation with MRST value of SLRT $(\mathrm{p}<0.05)$. Also, only the ADC parameter of the Lin-TD model and the CV parameter of the Lin-V model in SLRT moderately correlated with the $\mathrm{VO}_{2}$ max and distance values of YYIR1T ( $\mathrm{p}<0.05)$. There was a significant and high correlation between MMHRT in RTCD and HR in YYIR1T $(\mathrm{p}<0.05)$. However, it was determined that the $\mathrm{VO}_{2}$ max and distance values of YYIR1T had a significant and high correlation with the MRST value of RTCD $(p<0.05)$. There was no significant correlation between the parameters (CV and ADC) of Lin-TD and Lin-V models in RTCD with the YYIR1T parameters ( $p>0.05)$.

Table 3. Correlation analysis results between SLRT and RTCD parameters and YYIR1T parameters in young soccer players $(n=12)$

\begin{tabular}{|c|c|c|c|c|c|c|}
\hline \multirow[b]{2}{*}{ Test } & \multirow[b]{2}{*}{ Parameter } & \multirow[b]{2}{*}{ Correlation } & \multicolumn{4}{|c|}{ YYIR1T } \\
\hline & & & $\begin{array}{l}\mathrm{VO}_{2 \mathrm{max}} \\
(\mathrm{ml} / \mathrm{kg} / \mathrm{dk})\end{array}$ & $\begin{array}{c}\text { MAS } \\
(\mathrm{km} / \mathrm{h})\end{array}$ & $\begin{array}{l}\text { Distance } \\
(\mathrm{m})\end{array}$ & $\begin{array}{c}\mathrm{HR} \\
\text { (beat/min) }\end{array}$ \\
\hline \multirow{12}{*}{ SLRT } & \multirow{2}{*}{$\begin{array}{c}\text { MMHRT } \\
\text { (beat/min) }\end{array}$} & $r$ & 0.061 & -0.325 & 0.062 & 0.748 \\
\hline & & $p$ & 0.850 & 0.303 & 0.849 & $0.005^{*}$ \\
\hline & \multirow{2}{*}{$\begin{array}{l}\text { MRST } \\
(\mathrm{km} / \mathrm{h})\end{array}$} & $r$ & 0.889 & 0.479 & 0.889 & -0.011 \\
\hline & & $p$ & $0.001 *$ & 0.115 & $0.001^{*}$ & 0.973 \\
\hline & \multirow{2}{*}{$\begin{array}{c}\text { Lin-TD CV } \\
(\mathrm{km} / \mathrm{h})\end{array}$} & $r$ & 0.510 & 0.167 & 0.510 & -0.146 \\
\hline & & $p$ & 0.090 & 0.604 & 0.090 & 0.652 \\
\hline & \multirow{2}{*}{$\begin{array}{c}\operatorname{Lin}-T D \\
A D C(k m)\end{array}$} & $r$ & 0.686 & 0.495 & 0.687 & 0.139 \\
\hline & & $p$ & $0.014 *$ & 0.102 & $0.014 *$ & 0.667 \\
\hline & \multirow{2}{*}{$\begin{array}{c}\mathrm{Lin}-\mathrm{V} \text { CV } \\
(\mathrm{km} / \mathrm{h})\end{array}$} & $r$ & 0.589 & 0.276 & 0.590 & -0.149 \\
\hline & & $p$ & $0.044 *$ & 0.386 & $0.044 *$ & 0.644 \\
\hline & \multirow{2}{*}{$\underset{(\mathrm{km})}{\operatorname{Lin}-\mathrm{V} \text { ADC }}$} & $r$ & 0.338 & 0.244 & 0.338 & 0.233 \\
\hline & & $p$ & 0.283 & 0.444 & 0.283 & 0.466 \\
\hline
\end{tabular}




\begin{tabular}{|c|c|c|c|c|c|c|}
\hline \multirow[b]{2}{*}{ Test } & \multirow[b]{2}{*}{ Parameter } & \multirow[b]{2}{*}{ Correlation } & \multicolumn{4}{|c|}{ YYIR1T } \\
\hline & & & $\underset{\left(\mathrm{ml} / \mathrm{Kg}^{2} \mathrm{max} / \mathrm{dk}\right)}{\mathrm{V}^{2}}$ & $\begin{array}{c}\text { MAS } \\
(\mathrm{km} / \mathrm{h})\end{array}$ & $\begin{array}{l}\text { Distance } \\
(\mathrm{m})\end{array}$ & $\begin{array}{c}\mathrm{HR} \\
\text { (beat/min) }\end{array}$ \\
\hline \multirow{12}{*}{ RTCD } & \multirow{2}{*}{$\begin{array}{c}\text { MMHRT } \\
\text { (beat/min) }\end{array}$} & $r$ & -0.061 & -0.445 & -0.061 & 0.873 \\
\hline & & $p$ & 0.850 & 0.147 & 0.850 & $0.001 *$ \\
\hline & \multirow{2}{*}{$\begin{array}{l}\text { MRST } \\
(\mathrm{km} / \mathrm{h})\end{array}$} & $r$ & 0.735 & 0.333 & 0.735 & 0.092 \\
\hline & & $\mathrm{p}$ & $0.006 *$ & 0.290 & $0.006^{*}$ & 0.776 \\
\hline & \multirow{2}{*}{$\begin{array}{c}\text { Lin-TD CV } \\
(\mathrm{km} / \mathrm{h})\end{array}$} & $r$ & 0.545 & 0.123 & 0.546 & -0.097 \\
\hline & & $p$ & 0.067 & 0.703 & 0.067 & 0.765 \\
\hline & \multirow{2}{*}{$\begin{array}{c}\text { Lin-TD } \\
\text { ADC(km) }\end{array}$} & $r$ & -0.139 & 0.046 & -0.139 & 0.284 \\
\hline & & $p$ & 0.667 & 0.888 & 0.666 & 0.371 \\
\hline & \multirow{2}{*}{$\begin{array}{c}\mathrm{Lin}-\mathrm{V} \text { CV } \\
(\mathrm{km} / \mathrm{h})\end{array}$} & $r$ & 0.424 & -0.033 & 0.424 & 0.115 \\
\hline & & $\mathrm{p}$ & 0.169 & 0.918 & 0.169 & 0.722 \\
\hline & \multirow{2}{*}{$\begin{array}{l}\underset{\mathrm{Lin}-\mathrm{V} \text { ADC }}{(\mathrm{km})} \\
\text { (1) }\end{array}$} & $r$ & -0.028 & 0.233 & -0.028 & -0.015 \\
\hline & & $p$ & 0.931 & 0.466 & 0.930 & 0.964 \\
\hline
\end{tabular}

${ }^{*} p<0.05$, SLRT: Straight-line running tests, RTCD: Running tests with a change of direction, YYIR1T: Yo-Yo intermittent recovery level 1 test, MMHRT: Mean of the maximum heart rates in three tests, MRST: Mean running speed of three tests, Lin-TD CV: Critical velocity in the linear total distance model, Lin-TD ADC: Anaerobic distance capacity in the linear total distance model, Lin-V CV: Critical velocity in the linear velocity model, Lin-V ADC: Anaerobic distance capacity in the linear velocity model, $\mathrm{VO}_{2}$ max: Maximum oxygen uptake, MAS: Maximum aerobic speed, HR: Heart rate after test

\section{DISCUSSION}

The aim of the current study was to determine relationships between YYIR1T performance and the CV parameter determined by test protocols consisting of runs with a change of direction and straight runs. It was determined that MMHRT and MRST values in both SLRT and RTCD positively and significantly correlated with related parameters of YYIR1T (Table 3). In terms of the CV parameter, the Lin-V CV value moderately and significantly correlated with $\mathrm{VO}_{2}$ max and distance values of YYIR1T (Table 3). Also, it was found that MRST value of SLRT was higher than RTCD (Table 2). The change of direction requires deceleration and acceleration movements. Therefore, the energy demand is high in a change of direction. Similarly, the ADC value of both models was high in SLRT. This result indicated high energy demand of anaerobic activity with a change of direction. Messias et al. [30] found no significant correlation between lactate minimum speed and CV values in futsal players. The lactate value is an indicator of anaerobic endurance. In our study, the CV values had no correlation with the MAS value of YYIR1T. It might be said that there was a similarity between findings of the two studies. Also, Fairman et al. [31] found that the CV values of Lin-TD and Lin-V models correlated with relative $\mathrm{VO}_{2}$ max values of male soccer players. The CV value of the Lin-V model in SLRT had a moderate significant correlation with $\mathrm{VO}_{2}$ max values (Table 3 ). In RTCD, there was no significant correlation between $\mathrm{CV}$ in both models and $\mathrm{VO}_{2}$ max values. The runs with a change of direction might increase energy cost of running. However, the running velocity might decrease owing to turnings and motions with change of direction. These factors might affect the correlation between $\mathrm{CV}$ and $\mathrm{VO}_{2}$ max in YYIR1T.

Karsten et al. [12] reported that a significant increase was determined in the CV parameter in addition to the covered distance in YYIR1T after the eight-week strength training period. The parallel increase in YYIR1T and CV indicated the improvement in aerobic endurance of soccer players. Both parameters are indicators of aerobic endurance. Therefore, the aerobic fitness level of players might be followed by CV parameter. A moderate correlation between the distance covered in YYIR1T and CV in the Lin-V model was found in our study. This finding was similar to results of the mentioned study. Similarly to findings 
of Karsten et al. [12], Clark et al. [32] reported that the high intensity interval training significantly increased the running velocity at $\mathrm{VO}_{2} \max$ and $\mathrm{CV}$ of female soccer players. The positive effect on aerobic endurance of high intensity interval training was indicated in the mentioned study. It might be indicated that the parallel increase in both parameters showed the relationship between $\mathrm{CV}$ and $\mathrm{VO}_{2}$ max running velocity.

Florence and Weir [23] found a high correlation between marathon time and CV values. The marathon running is an aerobic activity and it dominantly uses the aerobic way of energy production. Similarly, Gamelin et al. [17] determined that one-hour running performance was related to the CV value. Also, Schnitzler et al. [33] found a high correlation between $\mathrm{CV}$ and the anaerobic threshold, maximal aerobic speed values. The relationship between YYIR1T performance and CV in the SLRT test is similar to the relationship in the mentioned studies. Aerobic endurance provides resistance to lactate accumulation in soccer matches. The high aerobic endurance capacity prevents high lactate accumulation in blood. Thus, the recovery capacity of players might be increased. The high recovery capacity allows performing intermittent high intensity activities without lactate accumulation in such sports as soccer. Thus, high intensity activities, such as sprint or runs with a change of direction might be performed more frequently in a match.

Guimarães and Da Silva [34] determined a positive correlation between CV and the anaerobic threshold running velocity. Also in the mentioned study, it was found a negative correlation between CV and duration of 3000 and $5000 \mathrm{~m}$ running. The anaerobic threshold is an indicator of aerobic endurance. The anaerobic threshold value is often used to determine athletes' aerobic endurance. Both CV and anaerobic threshold are important aerobic endurance parameters. Similarly, this relationship was sustained by a high negative correlation between $\mathrm{CV}$ and aerobic running durations in the mentioned study. The relationship between CV in SLRT and YYIR1T performance in the present study drew parallelism with findings of mentioned studies.

The critical power parameter was based on the power-exhaustion time relationship by Monod and Scherer [5]. The critical power tests were performed on a bike ergometer with different power values. The relationship between critical power and VO2max was indicated in many studies. Greco et al. [35] determined that the $\mathrm{VO}_{2}$ max value was highly correlated to critical power in both endurance cyclists and untrained subjects. The MRST value in SLRT and RTCD had a positive correlation with the $\mathrm{VO}_{2}$ max value in YYIR1T in the present study. The MRST was an indicator of loading intensity in running. The high mean running speed indicates the amount of anaerobic energy support in exercise. The high VO2max contributes this anaerobic energy support by developing recovery capacity. However, it was found that the VO2max value in YYIR1T had only correlation with CV value in SLRT. The SLRT had fewer runs with change of direction than RTCD and it was similar to YYIR1T. Running with a change of direction demands more energy, and fatigue may affect athletes more negatively during these runs. Therefore, it could be said that the $\mathrm{CV}-\mathrm{VO}_{2}$ max relationship could be affected by runs with change of direction.

The maximal lactate steady state is an important endurance parameter. The maximal lactate steady state was described as the highest exercise intensity that the blood lactate concentration had stability, and it related to endurance performance [36]. De Lucas et al. [37] reported that running velocity at maximal lactate steady state obtained by an intermittent test protocol was related to the CV value in endurance runners. It was found that the YYIR1T performance was related to CV value of the Lin-V model in SLRT. This finding drew parallelism to the results of the mentioned study. Also, Billat et al. [36] indicated that the mean running velocity during marathon was below the work load in the maximal lactate steady state. Similarly, Carter and Dekerle [38] found a positive 
correlation between the $\mathrm{VO}_{2}$ max value in a treadmill test and the CV value. In the light of these findings, it could be said that aerobic endurance performance could be evaluated by the CV parameter.

Dellal et al. [39] determined that physiological parameters in running with a change of direction were higher than straight line runs in soccer players. In the present study, the MRST value was lower in RTCD. The MRST indicated the exercise intensity during running. It was seen that the exercise intensity of SLRT was higher than RTCD. Players achieved a lower running speed in RTCD due to high physiological stress and this result was similar to findings in the mentioned study. It was indicated that activities with a change of direction could increase the dominance of anaerobic energy metabolism in exercise [39]. The physiological responses of exercise may be manipulated by activities with a change of directions. Particularly, the intermittent high intensity activities may be combined by runs with change of direction in soccer. However, this study was conducted on twelve elite young soccer players. Players' injuries were a limitation in this study. Therefore, injured players were not included in the research group. However, similar studies may be performed on professional players from different soccer leagues in next studies.

Kendall et al. [40] found a high positive correlation between the $\mathrm{CV}$ and $\mathrm{VO}_{2}$ max value in collegiate rowers. Rowing is an endurance sport, and the high aerobic endurance level (high $\mathrm{VO}_{2} \max$ ) is necessary for maximum performance in competitions. The recovery capacity in many sports depends on athletes' aerobic endurance level. The recovery duration between high intensity runs was shorter in soccer players having high aerobic endurance. The YYIR1T involves intermittent runs with turns and it reflects a running profile in soccer. Most of the recovery period between high intensity runs (runs at 16-19 $\mathrm{km} / \mathrm{h}$ speed interval and above $19 \mathrm{~km} / \mathrm{h}$ speed) consisted of 2-9 seconds (39.31\%) and 10 30 seconds (27.70\%) in elite level soccer matches [41]. The YYIR1T performance $\left(\mathrm{VO}_{2} \mathrm{max}\right.$ and covered distance) had a moderate correlation with the CV value of the Lin-V model in SLRT in our study. There was no correlation between YYIR1T parameters and the CV value in RTCD. The SLRT protocol involved less runs with change of direction than RTCD protocol. Therefore, it might be more similar to YYIR1T, and the correlation between two tests might have arisen from this similarity. The activities with a change of direction need more effort and energy. There might be no correlation between YYIR1T and CV parameters of RTCD for these reasons.

\section{CONCLUSION}

In summary, it might be said that the YYIR1T correlated with the CV parameter of the Lin-V model in SLRT. There was no significant correlation between CV in the Lin-TD model and YYIR1T performance. The SLRT contained less movements with a change of direction than RTCD. These activities with a change of direction may affect the relationship between test parameters and YYIR1T performance. The soccer game has variable movement patterns. The various high intensity activities (sprints, acceleration, change of direction, jumping, tackling) are frequently performed in soccer matches. These activities affect soccer performance and match scores. The performance of soccer specific activities should be developed with appropriate exercises. The CV parameter as an aerobic endurance indicator may be used to increase endurance performance of soccer players. The optimal aerobic endurance is fundamental of high anaerobic endurance level. The high $\mathrm{VO}_{2}$ max may delay oxygen debt during high intensity exercises. Therefore, the CV parameter obtained by the soccer specific test protocols, such as RTCD, may be used to monitor and develop aerobic endurance performance of soccer players. The CV obtained from different test protocols involving runs with a change of direction may be comparatively analyzed with performance of reference test protocols, such as YYIR1T. The CV test protocols containing runs with change of direction 
may be used for monitoring aerobic endurance of soccer players. Consequently, it may be said that runs with a change of direction may increase energy expenditure, and this phenomenon may cause lower estimation of ADC parameter in test protocols including runs with change of direction in contrast to similar estimation of the CV parameter.

\section{REFERENCES}

[1] Helgerud J, Engen LC, Wisloff U, Hoff J. Aerobic endurance training improves soccer performance. Med Sci Sport Exerc. 2001;33(11):1925-1931. https://doi.org/10.1097/00005768-200111000-00019

[2] Bangsbo J. Energy demands in competitive soccer. J Sport Sci. 1994;12(sup1):5-12. https://doi.org/10.1080/02640 414.1994.12059272

[3] Da Silva CD, Bloomfield J, Marins JCB. A review of stature, body mass and maximal oxygen uptake profiles of U17, U20 and first division players in Brazilian soccer. J Sport Sci Med. 2008;7(3):309.

[4] Dicks ND, Joe TV, Hackney KJ, Pettitt RW. Validity of critical velocity concept for weighted sprinting performance. Int J Exerc Sci. 2018;11(4):900.

[5] Monod H, Scherrer J. The work capacity of a synergic muscular group. Ergonomics. 1965;8(3):329-338. https://doi. org/10.1080/00140136508930810

[6] Ettema JH. Limits of human performance and energy-production. Internationale Zeitschrift Für Angewandte Physiologie Einschließlich Arbeitsphysiologie. 1966;2(1):45-54. https://doi.org/10.1007/bf00694796

[7] Hill DW. The critical power concept. Sport Med. 1993;16(4):237-254. https://doi.org/10.2165/00007256-19931604000003

[8] Overend TJ, Cunningham DA, Paterson DH, Smith WDF. Physiological responses of young and elderly men to prolonged exercise at critical power. Eur J Appl Physiolog Occup Physiol. 1992;64(2):187-193. https://doi.org/10.1007/bf00717959

[9] Jones AM, Vanhatalo A, Burnley M, Morton RH, Poole DC. Critical power: implications for determination of VO2max and exercise tolerance. Med Sci Sports Exerc. 2010; 42(10): 1876-90. https://doi.org/10.1249/mss.0b013e3181d9cf7f

[10] Walsh ML. Whole body fatigue and critical power. Sport Med. 2000;29(3):153-166. https://doi.org/10.2165/00007256200029030-00002

[11] Andrzejewski M, Pluta B, Konefał M, Konarski J, Chmura J, Chmura P. Activity profile in elite Polish soccer players. Res Sport Med. 2019; 27(4): 473-484. https://doi.org/10.1080/15438627.2018.1545648

[12] Karsten B, Larumbe-Zabala E, Kandemir G, Hazir T, Klose A, Naclerio F. The effects of a 6-week strength training on critical velocity, anaerobic running distance, 30-m sprint and Yo-Yo intermittent running test performances in male soccer players. PloS One. 2016;11(3):e0151448. https://doi.org/10.1371/journal.pone.0151448

[13] Lord C, Blazevich AJ, Abbiss CR, Ma'ayah F. Reliability and validity of maximal mean and critical speed and metabolic power in Australian youth soccer players. J Hum Kinetics. 2020;73(1):93-102. https://doi.org/10.2478/hukin-2019-0135

[14] Denadai BS, Gomide EBG, Greco CC. The relationship between onset of blood lactate accumulation, critical velocity, and maximal lactate steady state in soccer players. J Strength Cond Res. 2005;9(2):64-368. https://doi. org/10.1519/00124278-200505000-00021

[15] Krustrup P, Mohr M, Amstrup T, et al. The Yo-Yo intermittent recovery test: Physiological response, reliability, and validity. Med Sci Sport Exerc. 2003;35:697-705. https://doi.org/10.1249/01.MSS.0000058441.94520.32

[16] Bangsbo J, Iaia FM, Krustrup P. The Yo-Yo intermittent recovery test. Sport Med. 2008;38(1):37-51. https://doi. org/10.2165/00007256-200838010-00004

[17] Gamelin FX, Coquart J, Ferrari N, et al. Prediction of one-hour running performance using constant duration tests. J Strength Cond Res. 2006;20(4):735-739. https://doi.org/10.1519/00124278-200611000-00002

[18] Moritani T, Nagata A, Devries HA, Muro M. Critical power as a measure of physical work capacity and anaerobic threshold. Ergonomics. 1981;24(5):339-350. https://doi.org/10.1080/00140138108924856

[19] Bull AJ, Housh TJ, Johnson GO, Rana SR. Physiological responses at five estimates of critical velocity. Eur J Appl Physiol. 2008;102(6):711-720. https://doi.org/10.1007/s00421-007-0649-7

[20] Hill DW, Ferguson CS. A physiological description of critical velocity. Eur J Appl Physiol Occup Physiol. 1999;79(3):290293. https://doi.org/10.1007/s004210050509

[21] Gaesser GA, Carnevale TJ, Garfinkel A, Walter DO, Womack CJ. Estimation of critical power with nonlinear and linear models. Med Sci Sport Exerc. 1995; 7(10):1430-1438. https://doi.org/10.1249/00005768-199510000-00012

[22] Kranenburg KJ, Smith DJ. Comparison of critical speed determined from track running and treadmill tests in elite runners. Med Sci Sport Exerc. 1996;28(5):614-618. https://doi.org/10.1097/00005768-199605000-00013

[23] Florence SL, Weir JP. Relationship of critical velocity to marathon running performance. Eur J Appl Physiol Occup Physiol. 1997;75(3):274-278. https://doi.org/10.1007/s004210050160

[24] Housh TJ, Cramer JT, Bull AJ, Johnson GO, Housh DJ. The effect of mathematical modeling on critical velocity. European Journal of Applied Physiology. 2001; 84(5): 469-475. https://doi.org/10.1007/s004210000375

[25] Hughson RL, Orok CJ, Staudt LE. A high velocity treadmill running test to assess endurance running potential. International Journal of Sports Medicine. 1984;5(01):23-25. https://doi.org/10.1055/s-2008-1025875

[26] Whipp BJ, Huntsman DJ, Storer TW, Lamarra N, Wasserman K. A constant which determines the duration of tolerance to high-intensity work. Proceedings of Federation of American Societies for Experimental Biology. 1982;41(5):1591-1591.

[27] Poole DC, Ward SA, Gardner GW, Whipp BJ. Metabolic and respiratory profile of the upper limit for prolonged exercise in man. Ergonomics. 1988;31(9):1265-1279. https://doi.org/10.1080/00140138808966766

[28] Bull AJ, Housh TJ, Johnson GO, Perry SR. Effect of mathematical modeling on the estimation of critical power. Med Sci Sport Exerc. 2000;32(2):526. https://doi.org/10.1097/00005768-200002000-00040 
[29] Carter H, Pringle JS, Jones AM, Doust JH. Oxygen uptake kinetics during treadmill running across exercise intensity domains. Eur J Appl Physiol. 2002;86(4):347-354. https://doi.org/10.1007/s00421-001-0556-2

[30] Messias LHD, De Andrade VC, Rosante K, De Lima TB, Santa Cruz RAR, De Oliveira RM, De Barros Manchado-Gobatto F. Running anaerobic sprint test, lactate minimum and critical velocity protocol in shuttle futsal testing. Centr Eur J Sport Sci Med. 2015;12:5-15. https://doi.org/10.18276/cej.2015.4-01

[31] Fairman CM, Kendall KL, Hyde PN, Yarbrough MB, Rossi S, Sherman M, Fukuda D. Estimating and tracking changes in VO2max from a field-based critical velocity test in collegiate soccer players. J Sport Human Perf. 2015;3(1):1-10. https://doi.org/10.12922/jshp.0052.2015

[32] Clark IE, West BM, Reynolds SK, Murray SR, Pettitt RW. Applying the critical velocity model for an off-season interval training program. J Strength Cond Res. 2013;27(12):3335-3341. https://doi.org/10.1519/jsc.0b013e31828f9d87

[33] Schnitzler C, Heck G, Chatard JC, Ernwein V. A simple field test to assess endurance in inexperienced runners. J Strength Cond Res. 2010; 24(8): 2026-2031. https://doi.org/10.1519/jsc.0b013e3181d2c48d

[34] Guimarães MP, Silva S. Comparison between critical velocity and speed at the anaerobic threshold of moderately trained 5-km runners. J Phys Educ Sport. 2017;4:2437-2442. https://doi.org/10.7752/jpes.2017.04271

[35] Greco CC, Caritá RAC, Dekerle J, Denadai BS. Effect of aerobic training status on both maximal lactate steady state and critical power. Appl Physiol Nutr Metabolism. 2012;37(4):736-743. https://doi.org/10.1139/h2012-047

[36] Billat VL, Sirvent P, Py G, Koralsztein JP, Mercier J. The concept of maximal lactate steady state. Sport Med. 2003; 33(6):407-426. https://doi.org/10.2165/00007256-200333060-00003

[37] De Lucas RD, Dittrich N, Junior RB, De Souza KM, Guglielmo LGA. Is the critical running speed related to the intermittent maximal lactate steady state?. J Sport Sci Med. 2012;11(1):89.

[38] Carter H, Dekerle J. Metabolic stress at cycling critical power vs. running critical speed. Sci Sports 2014;29(1):51-54. https://doi.org/10.1016/j.scispo.2013.07.014

[39] Dellal A, Keller D, Carling C, Chaouachi A, Wong DP, Chamari K. Physiologic effects of directional changes in intermittent exercise in soccer players. J Strength Cond Res. 2010;24(12):3219-3226. https://doi.org/10.1519/jsc.0b013e$3181 \mathrm{~b} 94 \mathrm{a} 63$

[40] Kendall KL, Fukuda DH, Smith AE, Cramer JT, Stout JR. Predicting maximal aerobic capacity (VO2max) from the critical velocity test in female collegiate rowers. J Strength Cond Res. 2012;26(3): 733-738. https://doi.org/10.1519/ jsc.0b013e318225f3ac

[41] Vigne G, Gaudino C, Rogowski I, Alloatti G, Hautier C. Activity profile in elite Italian soccer team. International Journal of Sports Medicine. 2010; 31(05): 304-310. https://doi.org/10.1055/s-0030-1248320 